\title{
Microwave spectroscopy with vector network analyzer for interlayer exchange-coupled symmetrical and asymmetrical $\mathrm{NiFe} / \mathrm{Ru} / \mathrm{NiFe}$
}

\author{
M Belmeguenai ${ }^{1,2,4}$, T Martin ${ }^{1}$, G Woltersdorf ${ }^{1}$, G Bayreuther ${ }^{1}$, \\ V Baltz ${ }^{3}$, A K Suszka ${ }^{3}$ and B J Hickey ${ }^{3}$ \\ ${ }^{1}$ Institut für Experimentelle Physik, Universität Regensburg, Universitätsstraße 31, \\ D-93040 Regensburg, Germany \\ ${ }^{2}$ LPMTM, Institut Galilée, UPR 9001 du CNRS, Université Paris 13, 99 Avenue Jean-Baptiste \\ Clément, F-93430 Villetaneuse, France \\ ${ }^{3}$ School of Physics and Astronomy, E C Stoner Laboratory, University of Leeds, \\ Leeds LS2 9JT, UK \\ E-mail: belmegue@galilee.univ-paris13.fr
}

Received 24 January 2008, in final form 5 May 2008

Published 1 August 2008

Online at stacks.iop.org/JPhysCM/20/345206

\begin{abstract}
Vector network analyzer ferromagnetic resonance spectroscopy (VNA-FMR) is used here to study the different excited modes of sputtered asymmetrical $\mathrm{NiFe}(13.6 \mathrm{~nm}) / \mathrm{Ru}\left(\mathrm{d}_{\mathrm{Ru}}\right) /$

$\mathrm{NiFe}(27.2 \mathrm{~nm})$ exchange-coupled films with variable Ru thicknesses. The obtained results have been compared to those of the symmetrical $\mathrm{NiFe}(30 \mathrm{~nm}) / \mathrm{Ru}\left(\mathrm{d}_{\mathrm{Ru}}\right) / \mathrm{NiFe}(30 \mathrm{~nm})$. In both cases, the measurements show the existence of an optic and an acoustic precessional mode. The optic mode was only observed over limited field ranges, especially in the symmetrical trilayers. To overcome such a limitation, we developed a new technique similar to the longitudinal FMR, where the bias and the rf field are parallel to each other and perpendicular to the pinning field. Interestingly, and in contrast to the symmetrical trilayers, we observed a mode anti-crossing in the dispersion relation of the asymmetrical layers that we attributed to the thickness difference between the two NiFe layers. Our experimental results on the effect of the biquadratic coupling on the mode frequency variations are in good agreement with the theory.
\end{abstract}

(Some figures in this article are in colour only in the electronic version)

\section{Introduction}

Ferromagnetic bilayers exchange-coupled through a nonmagnetic layer are used in magnetoresistive read heads [1], magnetic recording devices and in toggled nonvolatile random access memory (MRAM) [2]. This toggled MRAM [2] has the advantage of a high switching field margin compared to the version using the Stoner-Wohlfarth scheme for writing. It requires an overlapping pulse sequence for switching, but increases the thermal stability of bits and reduces the dipolar coupling between bits [3]. However, the dynamics at $1-10 \mathrm{GHz}$

4 Author to whom any correspondence should be addressed. of these exchange-coupled layers, which determines the highspeed response, is a fundamental limit to the increasing data rates in such magnetic storage devices [4]. Therefore, understanding the nature and the extent of exchange interactions as well as their effect on the different excited modes in the $\mathrm{GHz}$ range is a technological key for such applications.

In prior work [5], we used a pulsed inductive microwave magnetometer (PIMM), conventional ferromagnetic resonance (FMR) and vector network analyzer FMR (VNA-FMR) for complementary studies of the dynamic properties of the symmetrical exchange-coupled $\mathrm{NiFe}(30 \mathrm{~nm}) / \mathrm{Ru}\left(\mathrm{d}_{\mathrm{Ru}}\right) / \mathrm{NiFe}$ $(30 \mathrm{~nm})$ films with variable $\mathrm{Ru}$ thicknesses $d_{\mathrm{Ru}}$. We focused our study on the effect of coupling on the characteristics 
of the different excited modes and we addressed the consequences of the nonlinear excitation resulting from the use of high amplitude and extremely short pulsed fields in such systems. The case of symmetrical exchange-coupled layers where the two ferromagnetic layers have the same thickness (here $30 \mathrm{~nm}$ ) is less complicated compared to the asymmetrical layers and, in contrast to the symmetrical layers, it has been far less explored [6-9]. In these works, Brillouin light scattering and FMR have been used to determine the coupling constants and anisotropies in $\mathrm{Co} / \mathrm{Cu} / \mathrm{Co}[6]$ and $\mathrm{Fe} / \mathrm{Cu} / \mathrm{Fe}$ [7] asymmetrical layers without addressing the effect of the thickness difference between ferromagnetic layers on the mode frequency. It is thus of great interest to see how this thickness difference between the two ferromagnetic coupled layers can affect the properties of the various modes. Therefore, the aim of this paper is to investigate the dynamic properties of asymmetrical $\mathrm{NiFe}(13.6 \mathrm{~nm}) / \mathrm{Ru}\left(\mathrm{d}_{\mathrm{Ru}}\right) / \mathrm{NiFe}(27.2 \mathrm{~nm})$ exchange-coupled films with variable $\mathrm{Ru}$ thicknesses $\left(d_{\mathrm{Ru}}\right)$ and to address their differences with those of the symmetrical trilayers $\mathrm{NiFe}(30 \mathrm{~nm}) / \mathrm{Ru}\left(\mathrm{d}_{\mathrm{Ru}}\right) / \mathrm{NiFe}(30 \mathrm{~nm})$ studied in detail in [5]. We focused our study on the difference in the mode amplitudes and frequencies as well as the effect of biquadratic coupling on the mode frequency. We thus took advantage of the VNA-FMR which, in contrast to FMR, allows us to follow the different excited modes over a large frequency range and on low applied bias fields not sufficient to saturate the specimens, to study these asymmetrical layers and to show the main differences with the symmetrical layers. Therefore, some prior results on the symmetrical systems [5] will be presented here for clarity of comparison. Moreover, we introduced and tested a new measurement procedure different from the conventional one usually used in VNA-FMR spectroscopy, allowing us to follow the optic mode frequency variations over a larger field range and thus increase the accuracy on coupling constants' estimation when fitting with theoretical models.

\section{Samples and experimental method}

The samples were prepared by dc magnetron sputtering. Two kinds of samples are used: (i) symmetrical layers: $\mathrm{Si} / \mathrm{Ta}(5 \mathrm{~nm}) / \mathrm{Ni}_{81} \mathrm{Fe}_{19}(30 \mathrm{~nm}) / \mathrm{Ru}\left(\mathrm{d}_{\mathrm{Ru}}\right) / \mathrm{Ni}_{81} \mathrm{Fe}_{19}(30 \mathrm{~nm}) / \mathrm{Ta}(5$ $\mathrm{nm})$ made in JENA and (ii) asymmetrical layers: $\mathrm{Si} / \mathrm{Ta}(4.5 \mathrm{~nm}) / \mathrm{Ni}_{81} \mathrm{Fe}_{19}(13.6 \mathrm{~nm}) / \mathrm{Ru}\left(\mathrm{d}_{\mathrm{Ru}}\right) / \mathrm{Ni}_{81} \mathrm{Fe}_{19}(27.2 \mathrm{~nm}) /$ $\mathrm{Ta}(4.5 \mathrm{~nm})$ made in Leeds. The Ru thickness is varied from 0.2 to $2.8 \mathrm{~nm}$ and from 0.13 to $4 \mathrm{~nm}$ for the symmetrical and asymmetrical layers, respectively. During the growth of the $\mathrm{Ni}_{81} \mathrm{Fe}_{19}$ layers, a magnetic field of 100 Oe was applied in an attempt to induce a uniaxial magnetic anisotropy with defined easy axis. The easy axes are parallel for both $\mathrm{Ni}_{81} \mathrm{Fe}_{19}$ layers.

For the dynamic measurements using VNA-FMR spectroscopy, the samples of $1 \times 1 \mathrm{~cm}^{2}$ are coupled to a coplanar waveguide; more details on the experimental set-up can be found in [10]. For the conventional configuration of measurement (see figure 1(c)), the data at each bias field $\left(H_{0}\right)$ require the subtraction of two measurements: one with the bias field switched on and a second measurement with a $1 \mathrm{kOe}$ saturating field applied in the same direction as the rf field $\left(h_{\mathrm{rf}}\right)$,
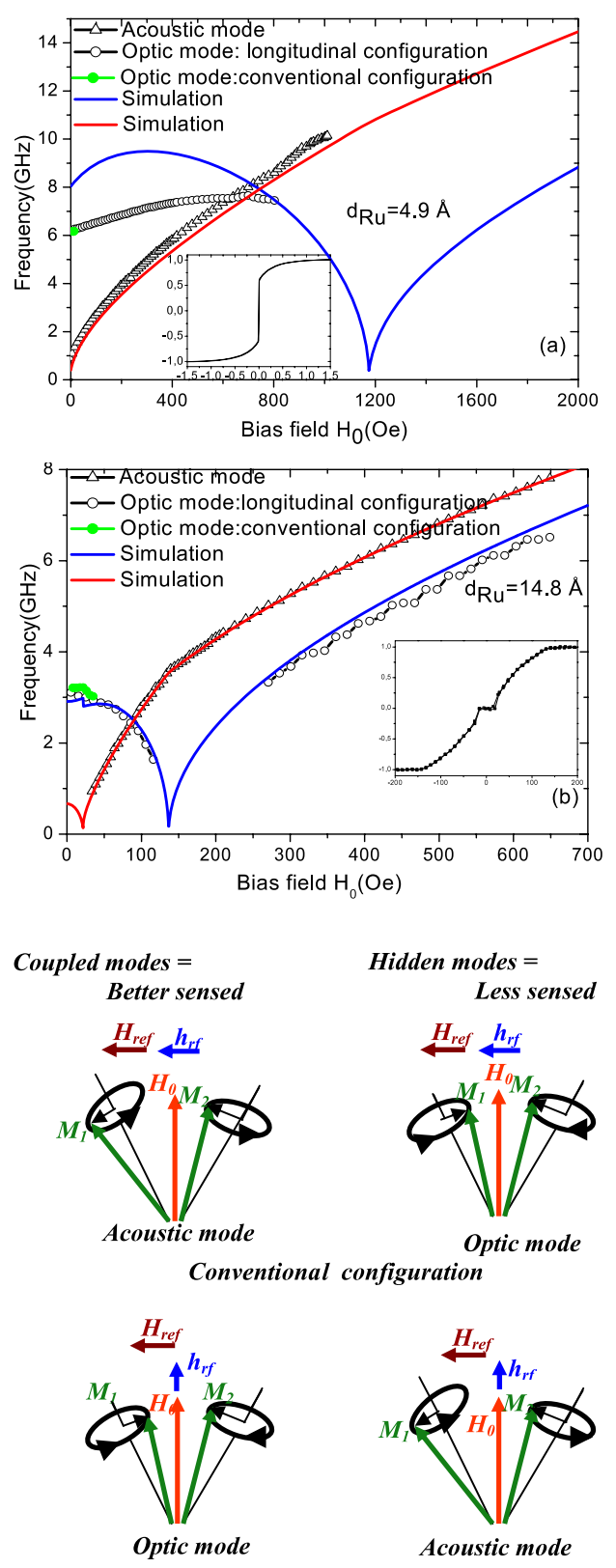

Longitudinal configuration

(c)

Figure 1. (a) and (b) Frequencies of the optic and acoustic modes of the coupled symmetrical $\mathrm{Si} / \mathrm{Ta} / \mathrm{NiFe}(30 \mathrm{~nm}) / \mathrm{Ru}\left(\mathrm{d}_{\mathrm{Ru}}\right) / \mathrm{NiFe}$ $(30 \mathrm{~nm}) / \mathrm{Ta}$ as a function of the easy axis bias field. These frequencies are obtained by fitting the module of the transmission coefficient $S_{21}$ measured. The corresponding simulations are obtained from the model of [13] using a uniaxial anisotropy field $H_{\text {ani }}=5$ Oe with (a) $J_{1}=-377 \mu \mathrm{J} \mathrm{m}^{-2}$ and $J_{2}=-514 \mu \mathrm{J} \mathrm{m}^{-2}$ and (b) $J_{1}=-140 \mu \mathrm{J} \mathrm{m}^{-2}$ and $J_{2}=-15 \mu \mathrm{J} \mathrm{m}^{-2}$. The inset shows the easy axis magnetization loop (normalized magnetization versus the static field in (a) kOe and (b) Oe). The green color (online) shows the optic mode frequency obtained using the conventional method of measurement described in section 2. (c) Geometry of the conventional and the longitudinal configurations of measurement. The developed configuration (longitudinal) was inspired from the longitudinal FMR technique $[8,9]$. In both configurations, we first apply a reference field $\left(H_{\text {ref }}\right)$ to saturate the sample magnetization and we acquire the signal. A bias field $H_{0}$ is then applied ( $\left.H_{\text {ref }}=0 \mathrm{Oe}\right)$ in a direction perpendicular to that where $H_{\text {ref }}$ has been applied and the signal is acquired. The data at each bias field are obtained from subtraction of the two measurements. 
which removes all magnetic response from the measured quantity (transmission coefficient: $S_{21}$ in dB). By subtracting this saturated measurement from the bias field measurement, all that remains is the effect of the oscillatory response induced by the precession of the magnetization in the sample. In our case, a maximum field of $1 \mathrm{kOe}$ was applied before each bias field measurement, in order to define an initial state. This field was reduced to the target bias field before the rf field was switched on. The resonance frequencies are obtained from the Lorentzian fit of the transmission characteristics measured by the VNA.

\section{Experimental results}

The interlayer exchange coupling between the magnetizations $M_{1}$ and $M_{2}$ of two ferromagnetic layers separated by a non-magnetic spacer layer is parameterized by the bilinear $\left(J_{1}\right)$ and biquadratic $\left(J_{2}\right)$ coupling parameters defined via the phenomenological energy density expression [11]:

$$
E=-J_{1} \frac{M_{1} \cdot M_{2}}{M_{1} M_{2}}-J_{2}\left(\frac{M_{1} \cdot M_{2}}{M_{1} M_{2}}\right)^{2} .
$$

The nature and the strength of the coupling are described by the signs and the magnitudes of $J_{1}$ and $J_{2}$.

Magneto-optical Kerr effect (MOKE) and vibrating sample magnetometer (VSM) were used at room temperature to obtain the hysteresis loops for each sample both in easy and hard axis directions. For antiferromagnetically (AF) coupled layers, the measured magnetization loops were then fitted numerically by minimizing the total energy of the system to determine the coupling constants $J_{1}$ and $J_{2}$ as described in [5]. The obtained results are shown in $[5,12]$ (for symmetrical trilayers).

In analogy with coupled harmonic oscillators, the precessional modes in two magnetic films coupled via a nonmagnetic interlayer can be classified into acoustic and optic modes, depending on whether the two film magnetizations precess in-phase or $180^{\circ}$ out-of-phase, respectively. The behavior of the mode frequencies as a function of applied fields provides extensive information about the magnitude and functional form of the coupling energy. The aim of this paper is to investigate the different precessional behavior of coupled symmetrical and asymmetrical layers. We first start by briefly presenting some results about the symmetrical layers. More details and results about these symmetrical layers can be found in [5]. Therefore, and in order to put in evidence the effect of the biquadratic coupling constant on the mode behavior, the typical experimental resonance frequencies for AF coupled symmetrical layers, over the whole range of spacer thicknesses, as a function of the external in-plane bias field $\left(H_{0}\right)$, are shown in figures 1(a) and (b) for two Ru thicknesses of 4.9 and $14.8 \AA$. For $d_{\mathrm{Ru}}=4.9 \AA$, both $J_{1}=-377 \mu \mathrm{J} \mathrm{m}^{-2}$ and $J_{2}=-514 \mu \mathrm{J} \mathrm{m}^{-2}$ are large and the AF coupling is strong while for $d_{\mathrm{Ru}}=14.8 \AA$ the AF coupling is weak and mainly $J_{1}=-140 \mu \mathrm{J} \mathrm{m}^{2}$ exists $\left(J_{2}=-15 \mu \mathrm{J} \mathrm{m}^{-2}\right)$. There are two different frequencies which appear in different field regimes. These modes are identified as the optic and acoustic precessional modes of the coupled ferromagnetic films. In contrast to figure 1(b), the experimental optic mode frequency in figure 1(a) increases slowly until it reaches a maximum around 600 Oe where again it starts to decrease. We found this behavior for all the samples with $4.3 \AA \leqslant d_{\mathrm{Ru}} \leqslant 11.2 \AA$, where the estimated $J_{2}$ is larger or comparable to $J_{1}$. The field, where the maximum of the optic mode frequency occurs, scales with the coupling strength. This behavior of the optic mode frequency reported in figure 1(a) is a consequence of the contribution of bilinear, biquadratic interlayer exchange and Zeeman energy to the effective stiffness of the magnetizations. It can be reproduced with a simple macro-spin model [13] using the values of $J_{1}$ and $J_{2}$ obtained from the fit of the VSM and the MOKE magnetization loops. The frequency offset of the optic mode in the simulation is possibly caused by the presence of a significant twisting of the magnetization along the film normal in the real sample. This additional effect can be treated by a multilayer simulation as shown by Buchmeier et al [14].

We note that for all AF coupled samples the optic mode was only observed over very limited field ranges using the conventional configuration of measurements described above (see figure 1(c)), especially in the symmetrical layers. In this configuration, the rf field $\left(h_{\mathrm{rf}}\right)$ is normal to the bias field $\left(H_{0}\right)$ direction. The magnetization components of each $\mathrm{NiFe}$ layer which are parallel to $h_{\mathrm{rf}}$ are added (resp. subtracted) to each other in the case of in-phase (resp. $180^{\circ}$ out-ofphase) precession. Therefore, the optic mode is less sensed in this configuration while the acoustic mode is better sensed (figure 1(c)) [8,9]. This makes the extraction of the coupling constants and the data analysis difficult. To overcome this difficulty and, basing on the longitudinal FMR technique [8, 9], we developed a new measurement configuration (longitudinal configuration in figure $1(\mathrm{c})$ ), which is more sensitive to the optic mode (see figures 1 and 4). Therefore, instead of applying $h_{\mathrm{rf}}$ perpendicular to $H_{0}$ direction, both fields were parallel to each other (figure 1(c)). This ensures that the magnetization components of each NiFe layer and which are parallel to the $h_{\mathrm{rf}}$ field are added to each other in the case of $180^{\circ}$ out-ofphase precession. Therefore, the optic mode is better sensed in this longitudinal configuration.

We note that in the two configurations (longitudinal and conventional) a reference field $\left(H_{\text {ref }}\right)$ is first applied in the direction perpendicular to that where the bias field is then applied (after switching off $H_{\text {ref }}$ ) and the data (in $\mathrm{dB}$ ) at each bias field $\left(H_{0}\right)$ are obtained after subtracting this reference measurement and that obtained when $H_{0}$ is switched on. As expected, this method allowed us to investigate the optic mode and to compare the measurements with the simulations over larger field ranges (figures 1 and 4).

Figure 2(a) shows an easy axis VSM room temperature magnetization loop of the asymmetrical $\mathrm{NiFe}(13.6 \mathrm{~nm}) / \mathrm{Ru}$ $(0.47 \mathrm{~nm}) / \mathrm{NiFe}(27.2 \mathrm{~nm})$ system with the corresponding simulation using the model described in [5]. The NiFe layers are antiferromagnetically coupled and their magnetizations are antiparallel at low magnetic field. When the magnetic field $H_{0}$ is applied along the easy axis, the $\mathrm{NiFe} / \mathrm{Ru} / \mathrm{NiFe}$ trilayer exhibits a spin-flop transition characteristic of a film in which there is a combination of AF coupling and uniaxial 

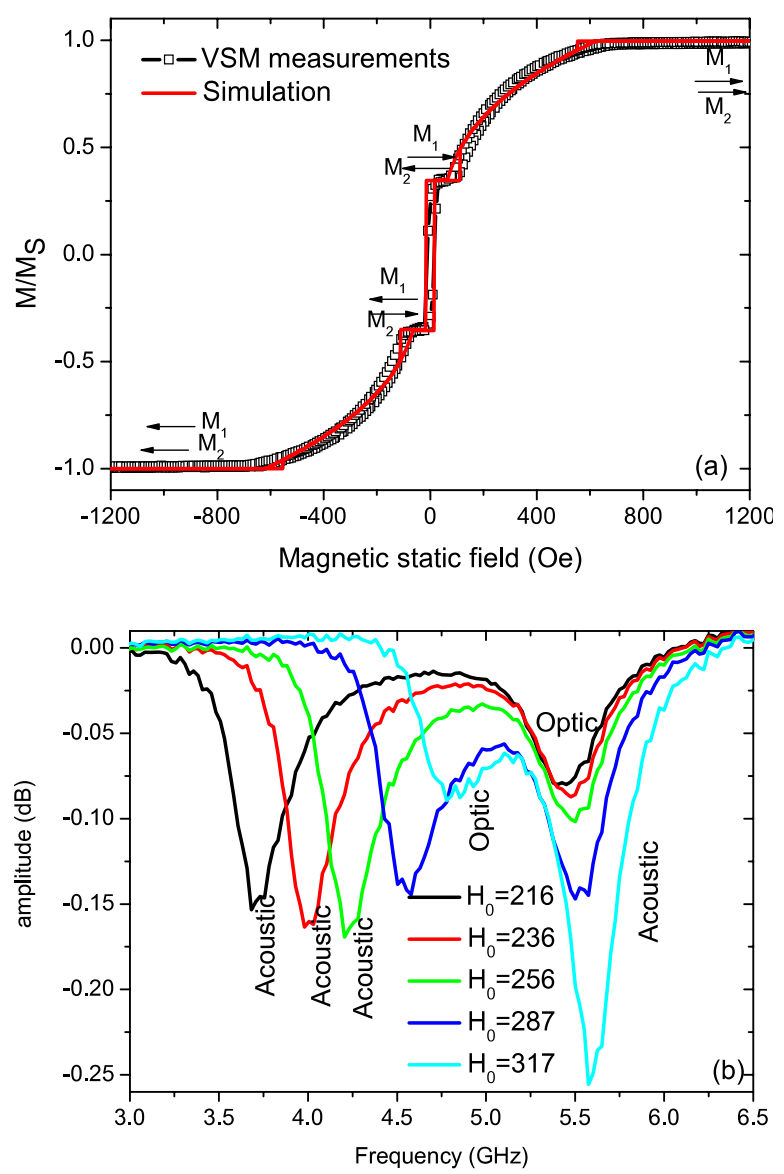

Figure 2. (a) Easy axis VSM magnetization loop and (b) amplitudes of the acoustic and optic modes as a function of the frequency for different applied static fields of the coupled asymmetrical $\mathrm{Si} / \mathrm{Ta} / \mathrm{NiFe}(13.6 \mathrm{~nm}) / \mathrm{Ru}(4.7 \AA) / \mathrm{NiFe}(27.2 \mathrm{~nm}) / \mathrm{Ta}$. The corresponding simulations for (a) are obtained from the models described in [5] with $J_{1}=-286 \mu \mathrm{J} \mathrm{m}^{-2}, J_{2}=-85 \mu \mathrm{J} \mathrm{m}^{-2}$ and uniaxial anisotropy constant $K_{\mathrm{u}}=200 \mathrm{~J} \mathrm{~m}^{-3}$. Arrows in (a) indicate the magnetization states for different applied fields.

anisotropy. This can be seen as discrete jumps in the simulated magnetization loop. At low fields $(H<65 \mathrm{Oe})$, the NiFe layers are AF aligned along the easy axis. In this phase, the component of the total magnetization of the trilayer, parallel to the field, equals one-third of the saturation magnetization (due to the thickness difference of NiFe layers). Higher fields induce a first-order phase transition in which the spins switch from being antiparallel along the easy axis to the spin-flop phase in which the spins reorient almost $90^{\circ}$ from the field direction but cant toward it.

Figure 2(b) represents the amplitudes of the corresponding signals to the optic and acoustic modes obtained in a $\mathrm{NiFe}(13.6 \mathrm{~nm}) / \mathrm{Ru}(0.47 \mathrm{~nm}) / \mathrm{NiFe}(27.2 \mathrm{~nm})$ trilayer using the conventional method of measurement for a different applied static field. For small applied fields, the optic mode has both higher frequency and amplitude (not shown). As the applied field magnitude increases, the acoustic mode amplitude increases and that of the optic mode decreases until we have a reversed situation (i.e. the acoustic mode has an amplitude which is several times higher than that the optic mode). When increasing again the applied field strength, the amplitude of the

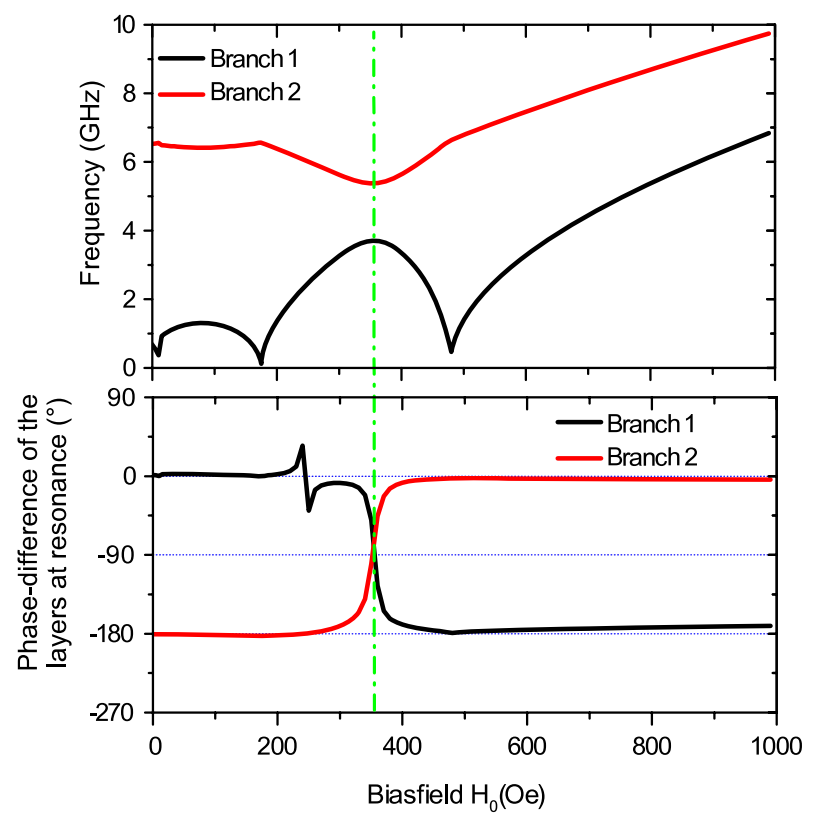

Figure 3. (Color online) Simulations of the excited mode frequencies and phase difference between the two ferromagnetic layers of the coupled asymmetrical $\mathrm{Si} / \mathrm{Ta} / \mathrm{NiFe}(13.6 \mathrm{~nm}) / \mathrm{Ru} / \mathrm{NiFe}(27.2 \mathrm{~nm}) / \mathrm{Ta}$ as a function of the easy axis bias field. The phase difference is calculated at the resonance frequency of each mode by linearization of the Landau-Lifshitz-Gilbert equation including the damping term $(\alpha=0.008)$ and for $J_{1}=-350 \mu \mathrm{J} \mathrm{m}^{-2}$ and $J_{2}=0 \mu \mathrm{J} \mathrm{m}^{-2}$. The two modes and the corresponding phases are referenced by branches 1 and 2 . The green vertical line refers to the field where the anti-crossing occurs. The $0^{\circ}$ phase difference corresponds to the acoustic mode and the $180^{\circ}$ refers to the optic mode.

acoustic mode decreases and that of the optic mode increases and the two modes have equal amplitudes at $H_{0}=287 \mathrm{Oe}$. The modes interchange then (for higher field) their positions (frequency) and amplitudes (i.e. the optic mode becomes the mode with a lower amplitude and frequency) without that their frequencies cross each other. The situation looks like each mode makes a jump to the position of the other and permutes its amplitude with the other mode. For higher applied field, the acoustic mode amplitude continues to increase and that of the optic mode decreases until they vanish and disappear (near the saturation).

This mode interchange is confirmed by the simulations represented in figure 3 showing the resonance frequencies for the different excited modes with the phase difference between the two magnetic NiFe layers at the resonance. These simulations are obtained for $\mathrm{NiFe}(13.6 \mathrm{~nm}) / \mathrm{Ru}(0.47 \mathrm{~nm}) / \mathrm{NiFe}(27.2 \mathrm{~nm})$ from the linearization of the Landau-Lifshitz-Gilbert equation including the damping term ( $\alpha=0.008$ [15]). In order to reduce the simulation complexity, the biquadratic coupling constant was neglected and we considered $J_{1}=-350 \mu \mathrm{J} \mathrm{m}^{-2}$ as a compromise between matching the saturation field and the optic mode frequency at zero field for this trilayer. The mode spectra show two different frequencies referenced by branches 1 and 2 . We note that the $0^{\circ}$ phase difference corresponds to the acoustic mode and the $180^{\circ}$ refers to the optic mode. Therefore, at low bias field $(<355 \mathrm{Oe})$ the optic mode has the higher frequency 


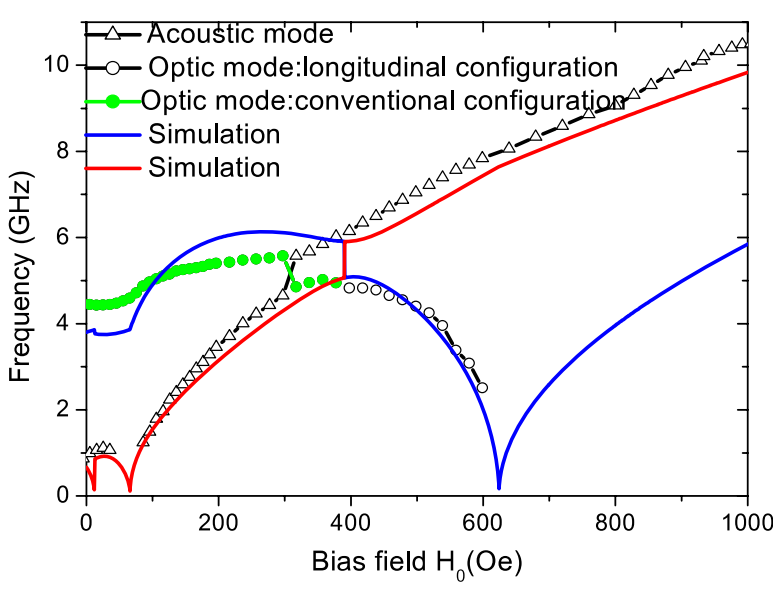

Figure 4. Frequencies of the optic and acoustic modes of the coupled asymmetrical Si/Ta/NiFe(13.6 nm)/Ru(4.7 $) / \mathrm{NiFe}$

$(27.2 \mathrm{~nm}) / \mathrm{T}$ as a function of the easy axis bias field. The corresponding simulations are obtained from the model of [13] with $J_{1}=-286 \mu \mathrm{J} \mathrm{m}^{-2}$ and $J_{2}=-85 \mu \mathrm{J} \mathrm{m}^{-2}$. The lower and upper frequency branches of each mode are joined by lines as a guide to the eye.

(branch 2) while the acoustic mode is at the lower frequency (branch 1). At $H_{0}=355 \mathrm{Oe}$, the change in the phase is traduced by a mode anti-crossing as explained above. The optic mode corresponds now $\left(H_{0}>355 \mathrm{Oe}\right)$ to branch 1 and the acoustic mode to branch 2 . For facility and practical reasons, the frequencies below and above the anti-crossing will be joined together in the figures to be presented below for each mode.

In the anti-crossing region (290 Oe $\left.<H_{0}<400 \mathrm{Oe}\right)$, the phase differences vary gradually as a function of the bias field (figure 3). We cannot thus assign the term 'acoustic' or 'optic' to each mode in this region since these modes correspond to a phase difference of $0^{\circ}$ or $180^{\circ}$, respectively, according to the definition of these terms given above. Therefore, we observe, close to the anti-crossing, something like 'hybrid modes' which are neither acoustic nor optic. Moreover, the kink in the phase difference at $H_{0}=245$ Oe is most probably caused by one magnetization rotating through the state where it is parallel to the rf field. Its phase with respect to the driving field experiences thus, at this point, a change by $180^{\circ}$, leading to a distortion of the phase difference.

We note that it is difficult to evaluate experimentally with accuracy the field value where the modes interchange and one needs to measure (with a well-calibrated VNA, for example) or to simulate the phase of the two modes. However, since the optic mode amplitude should vanish at high field near the saturation the decrease of the acoustic mode amplitude after reaching its maximal value remains a good indicator for the field range where the mode interchange occurs. In our case and for the experimental results to be presented below, we adopt the criterion that anti-crossing corresponds to the next bias field value where we have equal mode amplitudes.

The corresponding measured dispersion relation for this asymmetrical trilayer $(\mathrm{NiFe}(13.6 \mathrm{~nm}) / \mathrm{Ru}(0.47 \mathrm{~nm}) / \mathrm{NiFe}$ $(27.2 \mathrm{~nm}))$ is represented in figure 4 . It is the typical dispersion relation of the AF asymmetrical coupled layers seen

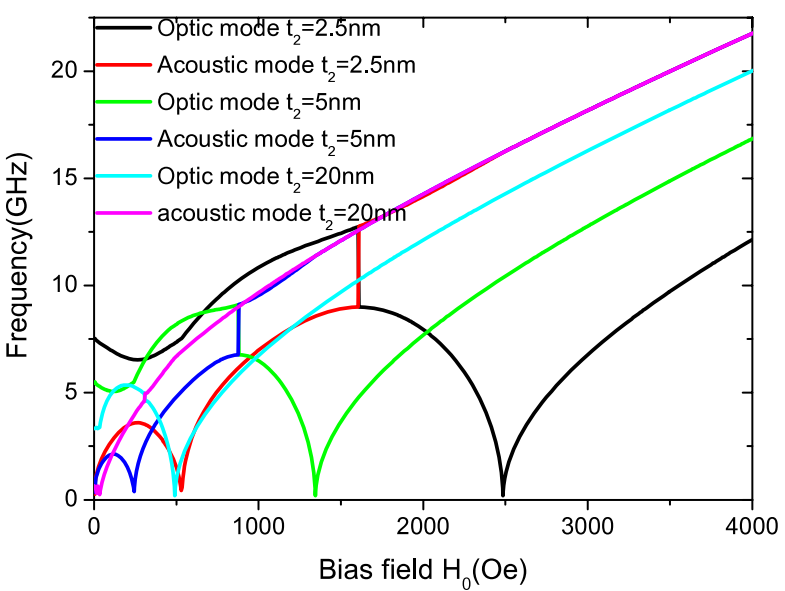

Figure 5. Simulated frequencies of the optic and acoustic modes of the coupled asymmetrical $\mathrm{Si} / \mathrm{Ta} / \mathrm{NiFe}\left(\mathrm{t}_{2}\right) / \mathrm{Ru}(4.7 \AA) / \mathrm{NiFe}$ $(27.2 \mathrm{~nm}) / \mathrm{Ta}$ as a function of the easy axis bias field for different thicknesses of the second NiFe layer. The corresponding simulations are obtained from the model of [13] with $J_{1}=-286 \mu \mathrm{J} \mathrm{m}^{-2}$ and $J_{2}=-85 \mu \mathrm{J} \mathrm{m}^{-2}$.

over the $\mathrm{Ru}$ thickness range. This variation of the mode frequencies with the external magnetic field relates to the different magnetic states of the two NiFe magnetizations. At very low fields $(0<H<65 \mathrm{Oe})$ the magnetizations align antiparallel to each other (see figure 2(a)). Therefore, both the optic mode, which has the higher frequency, and the acoustic mode frequencies are constant. In the spin-flop phase $(H>$ $65 \mathrm{Oe})$, the angle between the magnetizations continuously decreases and the optic mode frequency increases continuously as the magnetic field increases. This transition to the spin-flop phase manifests in a jump of both the optic and the acoustic mode frequency (at $65 \mathrm{Oe}$ ). For higher fields, while the acoustic mode frequency continues to increase, the optic mode frequency and intensity start to decrease before disappearing (figure 4). It should have a dip when the sample saturates at 625 Oe (see the simulation in figure 4). Although the mode frequency behavior is similar to the symmetrical layers, a significant difference occurs in the spin-flop range (around 300 Oe). The frequency branches show an anti-crossing, which was well reproduced by simulations in figure 4 . We are still investigating the origin of such an interchange and mode anticrossing. However, the fact that no jump or discontinuity is observed in the corresponding static magnetization loop shown in figure 2(a) suggests that this anti-crossing is just due to the difference in the thickness between the two NiFe layers. In fact, this difference of thickness breaks the symmetry of the system and thus lifts the degeneracy between the optic and acoustic mode frequencies whatever the magnetic field. According to our simulations shown in figure 5 using the model described in [13], the separation between the upper and the lower branch of the anti-crossing (which we call repulsion amplitude) decreases with the decreasing difference in thickness between the two magnetic layers when keeping constant all the magnetic parameters (coupling constants, magnetization at saturation, anisotropy, etc). It disappears when the two layers have the same thickness. This mode 


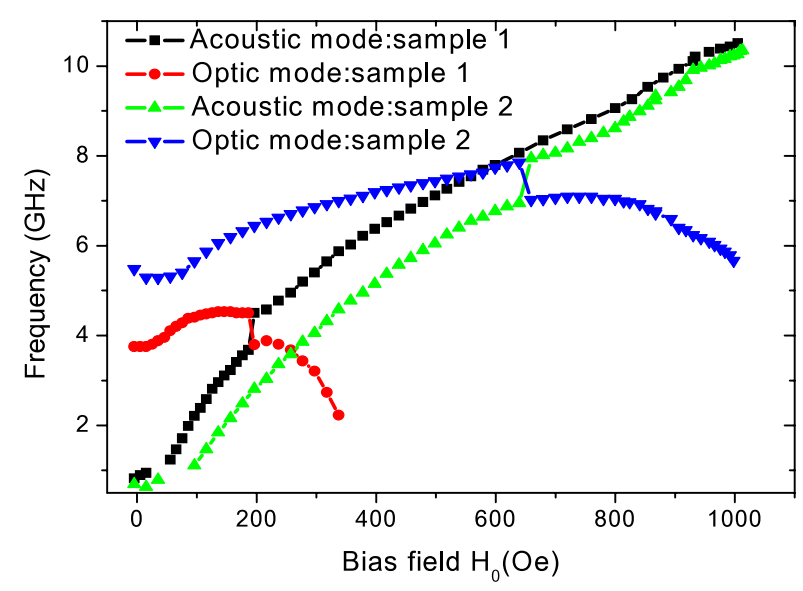

Figure 6. Frequencies of the optic and acoustic modes of the coupled asymmetrical Si/Ta/NiFe(13.6 nm)/Ru(5.18 $) / \mathrm{NiFe}$ $(27.2 \mathrm{~nm}) / \mathrm{Ta}$ (sample 1) and $\mathrm{Si} / \mathrm{Ta} / \mathrm{NiFe}(13.6 \mathrm{~nm}) / \mathrm{Ru}(4.23 \AA) / \mathrm{NiFe}$ $(27.2 \mathrm{~nm}) / \mathrm{Ta}$ (sample 2) as a function of the easy axis bias field. The corresponding coupling constants are $J_{1}=-180 \mu \mathrm{J} \mathrm{m}^{-2}$ and $J_{2}=-49 \mu \mathrm{J} \mathrm{m}^{-2}$ for sample 1 and $J_{1}=-526 \mu \mathrm{J} \mathrm{m}^{-2}$ and $J_{2}=-180 \mu \mathrm{J} \mathrm{m}^{-2}$ for sample 2 .

repulsion or anti-crossing has been seen experimentally for all asymmetrical AF coupled layers. Moreover, the repulsion amplitude is slightly coupling-dependent. It increases from $0.71 \mathrm{GHz}$ for trilayers with $J_{1}=-180 \mu \mathrm{J} \mathrm{m}^{-2}$ and $J_{2}=$ $-49 \mu \mathrm{J} \mathrm{m}^{-2}$ to $0.9 \mathrm{GHz}$ for trilayers with $J_{1}=-526 \mu \mathrm{J} \mathrm{m}^{-2}$ and $J_{2}=-180 \mu \mathrm{J} \mathrm{m}^{-2}$ as presented in figure 6 . These plots show that both the optic and acoustic mode frequencies are coupling-dependent below the saturation, as was discussed for the symmetrical layers in prior work [5].

\section{Conclusion}

The high frequency magnetization dynamics of interlayercoupled asymmetrical and symmetrical $\mathrm{NiFe} / \mathrm{Ru} / \mathrm{NiFe}$ films has been studied by VNA-FMR spectroscopy. We identified two modes observable over a short bias field range applied in the easy axis of the films. In order to access the optic mode over a large range of bias field, we developed a new technique similar to the longitudinal FMR, where the bias and rf field are parallel to each other and perpendicular to the pinning field. We showed that the variation of the mode frequencies with the external magnetic field relates to the different magnetic states of the two NiFe magnetizations. The mode anti-crossing that occurs in the dispersion relation of the asymmetrical layers has been attributed to the thickness difference between the two magnetic layers rather to any static magnetic state of the system. Further investigations are ongoing in order to better understand and quantify such an effect.

\section{References}

[1] Zhu J-G (Jimmy) 2003 Mater. Today 622

[2] Engel B N, Åkerman J, Butcher B, Dave R W, DeHerrera M, Durlam M, Grynkewich G, Janesky J, Pietambaram S V, Rizzo N D, Slaughter J M, Smith K, Sun J J and Tehrani S 2005 IEEE Trans. Magn. 41132

[3] Worledge D C 2006 IBM J. Res. Dev. 5069

[4] Back C H, Allenspach R, Weber W, Parkin S S P, Weller D, Garwin E L and Siegmann H C 1999 Science 285864

[5] Belmeguenai M, Martin T, Woltersdorf G and Bayreuther G 2007 Phys. Rev. B 76104414

[6] Heinrich B, Cochran J F, Kowalewski M, Kirschner J, Celinski Z, Arrott A S and Myrtle K 1991 Phys. Rev. B 449348

[7] Heinrich B, Celinski Z, Cochran J F, Muir W B, Rudd J, Zhong Q M, Arrott A S, Myrtle K and Kirschner J 1990 Phys. Rev. Lett. 64673

[8] Zhang Z, Zhou L, Wigen P E and Ounadjela K 1994 Phys. Rev. Lett. 73336

[9] Zhang Z, Zhou L, Wigen P E and Ounadjela K 1994 Phys. Rev. B 506094

[10] Neudecker I, Woltersdorf G, Heinrich B, Okuno T, Gubbiotti G and Back C H 2006 J. Magn. Magn. Mater. 307148

[11] Rührig M, Schäfer R, Hubert A, Mosler R, Wolf A J, Demokritov S O and Grünberg P 1991 Phys. Status Solidi a 125635

[12] Martin T, Belmeguenai M, Maier M, Perzlmaier K and Bayreuther G 2007 J. Appl. Phys. $10109 \mathrm{C} 101$

[13] Layadi A 2002 Phys. Rev. B 65104422

[14] Buchmeier M, Kuanr B K, Gareev R R, Bürgler D E and Grünberg P 2003 Phys. Rev. B 67184404

[15] Hiebert W K, Stankiewicz A and Freeman M R 1997 Phys. Rev. Lett. 791134 